\title{
A sum analogous to Dedekind sums and its hybrid mean value formula
}

\author{
by
}

WenPeng Zhang (Xi'an)

1. Introduction. For a positive integer $k$ and an arbitrary integer $h$, the classical Dedekind sum $S(h, k)$ is defined by

$$
S(h, k)=\sum_{a=1}^{k}\left(\left(\frac{a}{k}\right)\right)\left(\left(\frac{a h}{k}\right)\right),
$$

where

$$
((x))= \begin{cases}x-[x]-1 / 2 & \text { if } x \text { is not an integer, } \\ 0 & \text { if } x \text { is an integer. }\end{cases}
$$

The various properties of $S(h, k)$ were investigated by many authors. For example, T. M. Apostol [2] and L. Carlitz [3] obtained a reciprocity theorem of $S(h, k)$. J. B. Conrey et al. [5] studied the mean value distribution of $S(h, k)$, and first got an important asymptotic formula. The author [4] and [8] also studied some sums analogous to Dedekind sums, and proved several mean value theorems. In October, 2000, during his visit in Xi'an, Professor Todd Cochrane introduced a sum analogous to the Dedekind sum as follows:

$$
C(h, k)=\sum_{a=1}^{k}\left(\left(\frac{\bar{a}}{k}\right)\right)\left(\left(\frac{a h}{k}\right)\right),
$$

where $\bar{a}$ is defined by $a \bar{a} \equiv 1 \bmod k$ and $\sum_{a=1}^{\prime k}$ denotes the summation over all $1 \leq a \leq k$ such that $(a, k)=1$. Then he suggested studying the arithmetical properties and mean value distribution properties of $C(h, k)$. Concerning these problems, we have not made any progress yet. But for a square-full number $k$ (i.e. $p \mid k$ if and only if $p^{2} \mid k$ ), the author [7] found that there are some close relations between $C(h, k)$ and the classical Kloosterman sum

2000 Mathematics Subject Classification: Primary 11L05.

Key words and phrases: sums analogous to Dedekind sum, mean value, asymptotic formula.

This work is supported by the N.S.F. and P.N.S.F. of P.R. China. 


$$
K(m, n, k)=\sum_{b=1}^{k} e^{\prime} e\left(\frac{m b+n \bar{b}}{k}\right),
$$

where $e(y)=e^{2 \pi i y}$, and proved the following asymptotic formula:

$$
\sum_{h=1}^{k} K(h, 1 ; k) C(h, k)=\frac{-1}{2 \pi^{2}} k \phi(k)+O\left(k \exp \left(\frac{3 \ln k}{\ln \ln k}\right)\right),
$$

where $\exp (y)=e^{y}$.

In this paper, we shall discuss the hybrid mean value problem involving $C(h, k)$ and the general Kloosterman sum

$$
K(m, n, r ; k)=\sum_{b=1}^{k} e\left(\frac{m b^{r}+n \bar{b}^{r}}{k}\right),
$$

where $r$ is any fixed positive integer. We shall use estimates for character sums and the mean value theorem of Dirichlet $L$-functions to prove the following:

THEOREM 1. Let $p$ be an odd prime. Then we have the asymptotic formula

$$
\sum_{h=1}^{p-1} K(h, 1 ; p) C(h, p)=\frac{-1}{2 \pi^{2}} p^{2}+O\left(p \exp \left(\frac{3 \ln p}{\ln \ln p}\right)\right) .
$$

TheOREM 2. Let $p$ be an odd prime. Then for any positive integer $r \geq 2$,

$$
\sum_{h=1}^{p-1} K(h, 1, r ; p) C(h, p)=\frac{-1}{2 \pi^{2}} p^{2}+O\left(r p^{3 / 2} \ln ^{2} p\right) .
$$

From Theorem 2 we may immediately deduce the following:

Corollary. Let $p$ be an odd prime. Then for any fixed $\varepsilon>0$, the asymptotic formula

$$
\sum_{h=1}^{p-1} K(h, 1, r ; p) C(h, p) \sim \frac{-1}{2 \pi^{2}} p^{2} \quad \text { as } p \rightarrow \infty
$$

holds for all integer $2 \leq r \leq p^{1 / 2-\varepsilon}$.

For general integer $k>2$, it is an unsolved problem whether there exists an asymptotic formula for $\sum_{h=1}^{\prime k} K(h, 1, r ; k) C(h, k)$. We conjecture that

$$
\sum_{h=1}^{k} K(h, 1, r ; k) C(h, k) \sim \frac{-1}{2 \pi^{2}} k \phi(k) \quad \text { as } k \rightarrow \infty
$$

for all integer $k>2$ and any fixed positive integer $r$. 
2. Some lemmas. We need the following lemmas:

Lemma 1 (see [7]). Let $a, k$ be integers with $k \geq 3$ and $(a, k)=1$. Then

$$
C(a, k)=\frac{-1}{\pi^{2} \phi(k)} \sum_{\substack{\chi \bmod k \\ \chi(-1)=-1}} \bar{\chi}(a)\left(\sum_{n=1}^{\infty} \frac{G(\chi, n)}{n}\right)^{2},
$$

where $\chi$ runs through the Dirichlet characters modulo $k$ with $\chi(-1)=-1$, and

$$
G(\chi, n)=\sum_{b=1}^{k} \chi(b) e\left(\frac{b n}{k}\right)
$$

denotes the Gauss sum corresponding to $\chi$.

Lemma 2 (see [7]). Let $k>2$ be any integer. Then

$$
\sum_{\substack{\chi \bmod k \\ \chi(-1)=-1}} L^{2}(1, \chi)=\frac{1}{2} \phi(k)+O\left(\exp \left(\frac{3 \ln k}{\ln \ln k}\right)\right) .
$$

Lemma 3. Let $k>2$ be any integer. Then

$$
\sum_{\substack{1 \leq a \leq k \\(a, k)=1}}\left|\sum_{\substack{\chi \bmod k \\ \chi(-1)=-1}} \chi(a) L^{2}(1, \bar{\chi})\right| \ll k \ln ^{2} k .
$$

Proof. Let $\tau(n)$ be the Dirichlet divisor function. Then for any $N \geq k$ and non-principal character $\chi$ modulo $k$, applying Abel's identity we obtain

$$
L^{2}(1, \chi)=\sum_{n=1}^{\infty} \frac{\chi(n) \tau(n)}{n}=\sum_{1 \leq n \leq N} \frac{\chi(n) \tau(n)}{n}+\int_{N}^{\infty} \frac{A(y, \chi)}{y^{2}} d y,
$$

where

$$
A(y, \chi)=\sum_{N<n \leq y} \chi(n) \tau(n) .
$$

From (1) we have

$$
\begin{aligned}
& \sum_{\substack{1 \leq a \leq k \\
(a, k)=1}}\left|\sum_{\substack{\chi \bmod k \\
\chi(-1)=-1}} \chi(a) L^{2}(1, \bar{\chi})\right| \\
& \leq \sum_{\substack{1 \leq a \leq k \\
(a, k)=1}}\left|\sum_{\substack{\chi \bmod k \\
\chi(-1)=-1}} \sum_{1 \leq n \leq N} \frac{\tau(n) \chi(a) \bar{\chi}(n)}{n}\right| \\
& \\
& \quad+\sum_{\substack{1 \leq a \leq k \\
(a, k)=1}}\left|\sum_{\substack{\chi \bmod k \\
\chi(-1)=-1}} \chi(a) \int_{N}^{\infty} \frac{A(y, \bar{\chi})}{y^{2}} d y\right| \equiv M_{1}+M_{2} .
\end{aligned}
$$


Now we estimate $M_{1}$ and $M_{2}$ in (2) respectively. Note that for $(a, k)=1$, from the orthogonality relation for characters we have

$$
\begin{aligned}
\sum_{\substack{\chi \bmod k \\
\chi(-1)=-1}} \chi(a) & =\frac{1}{2} \sum_{\chi \bmod k}(1-\chi(-1)) \chi(a) \\
& =\frac{1}{2} \sum_{\chi \bmod k} \chi(a)-\frac{1}{2} \sum_{\chi \bmod k} \chi(-a) \\
& = \begin{cases}\frac{1}{2} \phi(k) & \text { if } a \equiv 1(\bmod k), \\
-\frac{1}{2} \phi(k) & \text { if } a \equiv-1(\bmod k), \\
0 & \text { otherwise. }\end{cases}
\end{aligned}
$$

Applying (3) we can get the estimate

$$
\text { (4) } \begin{aligned}
M_{1} & =\sum_{\substack{1 \leq a \leq k \\
(a, k)=1}}\left|\frac{1}{2} \phi(k) \sum_{\substack{1 \leq n \leq N \\
n \equiv a(\bmod k)}}^{\prime} \frac{\tau(n)}{n}-\frac{1}{2} \phi(k) \sum_{\substack{1 \leq n \leq N \\
n \equiv-a(\bmod k)}}^{\prime} \frac{\tau(n)}{n}\right| \\
& \leq \frac{1}{2} \phi(k) \sum_{\substack{1 \leq a \leq k \\
(a, k)=1}} \sum_{\substack{1 \leq n \leq N \\
n \equiv a(\bmod k)}}^{\prime} \frac{\tau(n)}{n}+\frac{1}{2} \phi(k) \sum_{\substack{1 \leq a \leq k \\
(a, k)=1}} \sum_{\substack{1 \leq n \leq N \\
n \equiv-a(\bmod k)}}^{\prime} \frac{\tau(n)}{n} \\
& \leq \phi(k) \sum_{1 \leq n \leq N}^{\prime} \frac{\tau(n)}{n} \ll \phi(k) \ln ^{2} N .
\end{aligned}
$$

Applying Cauchy's inequality and estimates for character sums,

$$
\begin{aligned}
\sum_{\chi \neq \chi_{0}}\left|\sum_{N \leq n \leq M} \chi(n)\right|^{2} & =\sum_{\chi \neq \chi_{0}}\left|\sum_{N \leq n \leq M \leq N+d} \chi(n)\right|^{2} \\
& =\phi(d) \sum_{N \leq n \leq M \leq N+d} \chi_{0}(n)-\left|\sum_{N \leq n \leq M \leq N+d} \chi_{0}(n)\right|^{2} \\
& \leq \frac{\phi^{2}(d)}{4},
\end{aligned}
$$

we have

$$
\begin{aligned}
\sum_{\substack{\chi \bmod k \\
\chi(-1)=-1}}|A(y, \chi)| \ll & \sqrt{\phi(k)}\left(\sqrt{y} \sum_{n \leq \sqrt{y}} \sum_{\substack{\chi \bmod k \\
\chi(-1)=-1}}\left|\sum_{m \leq y / n} \chi(m)\right|^{2}\right)^{1 / 2} \\
& +\sum_{\substack{\chi \bmod k \\
\chi(-1)=-1}}\left|\sum_{n \leq \sqrt{y}} \chi(n)\right|^{2}+\sum_{\substack{\chi \bmod k \\
\chi(-1)=-1}}\left|\sum_{n \leq \sqrt{N}} \chi(n)\right|^{2} \\
& \ll \sqrt{y} \sqrt{\phi^{3}(k)} .
\end{aligned}
$$


From (5) we can also get the estimate

$$
\begin{aligned}
M_{2} & =\sum_{\substack{1 \leq a \leq k \\
(a, k)=1}}\left|\sum_{\substack{\chi \bmod k \\
\chi(-1)=-1}} \chi(a) \int_{N}^{\infty} \frac{A(y, \bar{\chi})}{y^{2}} d y\right| \\
& \leq \sum_{\substack{1 \leq a \leq k \\
(a, k)=1}} \int_{N}^{\infty} \frac{1}{y^{2}} \sum_{\substack{\chi \bmod k \\
\chi(-1)=-1}}|A(y, \bar{\chi})| d y \\
& \ll \frac{\phi^{5 / 2}(k)}{\sqrt{N}} .
\end{aligned}
$$

Taking $N=k^{3}$, combining (4), (5) and (6) we immediately get the estimate

$$
\sum_{\substack{1 \leq a \leq k \\(a, k)=1}}\left|\sum_{\substack{\chi \bmod k \\ \chi(-1)=-1}} \chi(a) L^{2}(1, \bar{\chi})\right| \ll k \ln ^{2} k .
$$

This proves Lemma 3.

3. Proof of Theorems 1 and 2. In this section, we complete the proof of Theorems 1 and 2. Let $p$ be an odd prime. Then from Lemma 1 and the properties of Gauss sums (see Theorem 8.19 of [1]) we can get the identity

$$
\begin{aligned}
C(a, p) & =\frac{-1}{\pi^{2} \phi(p)} \sum_{\substack{\chi \bmod p \\
\chi(-1)=-1}} \bar{\chi}(a)\left(\sum_{n=1}^{\infty} \frac{G(\chi, n)}{n}\right)^{2} \\
& =\frac{-1}{\pi^{2} \phi(p)} \sum_{\substack{\chi \bmod p \\
\chi(-1)=-1}} \bar{\chi}(a) \tau^{2}(\chi) L^{2}(1, \bar{\chi}) .
\end{aligned}
$$

For any fixed positive integer $r$, applying (7) we deduce

$$
\begin{aligned}
& \sum_{h=1}^{p-1} K(h, 1, r ; p) C(h, p) \\
& \quad=\frac{-1}{\pi^{2} \phi(p)} \sum_{\substack{\chi \bmod p \\
\chi(-1)=-1}}\left(\sum_{h=1}^{p-1} \bar{\chi}(h) K(h, 1, r ; p)\right) \tau^{2}(\chi) L^{2}(1, \bar{\chi}) .
\end{aligned}
$$

For any primitive character $\chi$ modulo $p$, from the properties of Gauss sums we have

$$
\tau(\chi) \tau(\bar{\chi})=-p \quad \text { if } \chi(-1)=-1
$$


and

$$
\begin{aligned}
\sum_{h=1}^{p-1} \bar{\chi}(h) K(h, 1, r ; p) & =\sum_{b=1}^{p-1} \sum_{h=1}^{p-1} \bar{\chi}(h) e\left(\frac{h b^{r}+\bar{b}^{r}}{p}\right) \\
& =\sum_{b=1}^{p-1} \chi\left(b^{r}\right) e\left(\frac{\bar{b}^{r}}{p}\right) \sum_{h=1}^{p-1} \bar{\chi}(h) e\left(\frac{h}{p}\right) \\
& =\tau(\bar{\chi}) G\left(1, \bar{\chi}^{r}, r ; p\right) .
\end{aligned}
$$

Noting $G(1, \chi, 1 ; p)=\tau(\chi)$, from $(8)$, (9), and Lemma 2 we immediately obtain

$$
\begin{aligned}
\sum_{h=1}^{p-1} K(h, 1 ; p) C(h, p) & =\frac{-1}{\pi^{2} \phi(p)} \sum_{\substack{\chi \bmod p \\
\chi(-1)=-1}} \tau^{2}(\bar{\chi}) \tau^{2}(\chi) L^{2}(1, \bar{\chi}) \\
& =\frac{-1}{\pi^{2}} \cdot \frac{p^{2}}{\phi(p)} \sum_{\substack{\chi \bmod p \\
\chi(-1)=-1}} L^{2}(1, \bar{\chi}) \\
& =\frac{-1}{2 \pi^{2}} p^{2}+O\left(p \exp \left(\frac{3 \ln p}{\ln \ln p}\right)\right) .
\end{aligned}
$$

This completes the proof of Theorem 1 .

Now we prove Theorem 2. Note that

$$
\begin{aligned}
G\left(1, \bar{\chi}^{r}, r ; p\right) \tau(\chi) & =\sum_{a=1}^{p-1} \sum_{b=1}^{p-1} \chi(a) \bar{\chi}^{r}(b) e\left(\frac{a+b^{r}}{p}\right) \\
& =\sum_{a=1}^{p-1} \sum_{b=1}^{p-1} \chi(a) e\left(\frac{a b^{r}+b^{r}}{p}\right) \\
& =\sum_{a=1}^{p-1} \chi(a)\left(\sum_{b=1}^{p-1} e\left(\frac{(a+1) b^{r}}{p}\right)\right)
\end{aligned}
$$

and

$$
\left|\sum_{b=1}^{p-1} e\left(\frac{n b^{r}}{p}\right)\right| \leq r \sqrt{p} \quad \text { for all integer } n \text { with }(p, n)=1 .
$$

(This result follows from Weil's general upper bound on exponential sums, see $[6]$.

From (8), (9), Lemma 2 and Lemma 3 we have

$$
\sum_{h=1}^{p-1} K(h, 1, r ; p) C(h, p)=\frac{-1}{\pi^{2} \phi(p)} \sum_{\substack{\chi \bmod p \\ \chi(-1)=-1}} \tau(\bar{\chi}) G\left(1, \bar{\chi}^{r}, r ; p\right) \tau^{2}(\chi) L^{2}(1, \bar{\chi})
$$




$$
\begin{aligned}
& =\frac{p}{\pi^{2} \phi(p)} \sum_{\substack{\chi \bmod p \\
\chi(-1)=-1}} G\left(1, \bar{\chi}^{r}, r ; p\right) \tau(\chi) L^{2}(1, \bar{\chi}) \\
& =\frac{p}{\pi^{2} \phi(p)} \sum_{a=1}^{p-1}\left(\sum_{b=1}^{p-1} e\left(\frac{(a+1) b^{r}}{p}\right)\right) \sum_{\substack{\chi \bmod p \\
\chi(-1)=-1}} \chi(a) L^{2}(1, \bar{\chi}) \\
& =\frac{-p(p-1)}{\pi^{2} \phi(p)} \sum_{\substack{\chi \bmod p \\
\chi(-1)=-1}} L^{2}(1, \bar{\chi}) \\
& +\frac{p}{\pi^{2} \phi(p)} \sum_{a=1}^{p-2}\left(\sum_{b=1}^{p-1} e\left(\frac{(a+1) b^{r}}{p}\right)\right) \sum_{\substack{\chi \bmod p \\
\chi(-1)=-1}} \chi(a) L^{2}(1, \bar{\chi}) \\
& =\frac{-1}{2 \pi^{2}} p^{2}+O\left(p \exp \left(\frac{3 \ln p}{\ln \ln p}\right)\right) \\
& +O\left(\sum_{a=1}^{p-2}\left|\sum_{b=1}^{p-1} e\left(\frac{(a+1) b^{r}}{p}\right)\right| \cdot\left|\sum_{\substack{\chi \bmod p \\
\chi(-1)=-1}} \chi(a) L^{2}(1, \bar{\chi})\right|\right) \\
& =\frac{-1}{2 \pi^{2}} p^{2}+O\left(r p^{3 / 2} \ln ^{2} p\right) .
\end{aligned}
$$

This proves Theorem 2 .

Acknowledgments. The author express his gratitude to the referee for his very helpful and detailed comments.

\section{References}

[1] T. M. Apostol, Introduction to Analytic Number Theory, Springer, New York, 1976.

[2] - Modular Functions and Dirichlet Series in Number Theory, Springer, New York, 1976.

[3] L. Carlitz, The reciprocity theorem of Dedekind sums, Pacific J. Math. 3 (1953), 513-522.

[4] X. F. Chen and W. P. Zhang, A sum analogous to Dedekind sums and its mean value formula, Chinese Ann. Math. 21 (2000), 715-722.

[5] J. B. Conrey, E. Fransen, R. Klein and C. Scott, Mean values of Dedekind sums, J. Number Theory 56 (1996), 214-226.

[6] A. Weil, On some exponential sums, Proc. Nat. Acad. Sci. U.S.A. 34 (1948), 203-210.

[7] W. P. Zhang, On a Cochrane sum and its hybrid mean value formula, J. Math. Anal. Appl. 267 (2002), 89-96. 
[8] W. P. Zhang and Y. Yi, On the 2m-th power mean of certain Hardy sums, Soochow J. Math. 26 (2000), 73-84.

Research Center for Basic Science

Xi'an Jiaotong University

Xi'an, Shaanxi, P.R. China

E-mail: wpzhang@nwu.edu.cn

Received on 19.2.2001

and in revised form on 3.4.2002 